Revue de l'Institut des langues et cultures

d'Europe, Amérique, Afrique, Asie et Australie

37 | 2019

Des genres en Méditerranée : pratiques,

représentations et transfert

\title{
Après les « Printemps arabes » : genres du discours touristique et argumentation
}

After the "Arab Spring": Speech Gendres of Tourism Discourse and Argumentation

Nabil Sukkari

URL : http://journals.openedition.org/ilcea/7679

DOI : 10.4000/ilcea.7679

ISSN : 2101-0609

Éditeur

UGA Éditions/Université Grenoble Alpes

Édition imprimée

ISBN : 978-2-37747-099-0

ISSN : $1639-6073$

Référence électronique

Nabil Sukkari, «Après les « Printemps arabes » : genres du discours touristique et argumentation », ILCEA [En ligne], 37 | 2019, mis en ligne le 04 novembre 2019, consulté le 10 décembre 2020. URL : http://journals.openedition.org/ilcea/7679 ; DOI : https://doi.org/10.4000/ilcea.7679

Ce document a été généré automatiquement le 10 décembre 2020.

(C) ILCEA 


\title{
Après les « Printemps arabes » : genres du discours touristique et argumentation
}

\author{
After the "Arab Spring": Speech Gendres of Tourism Discourse and \\ Argumentation
}

Nabil Sukkari

\section{Introduction}

Le tourisme est un mode de médiation entre les cultures qui ne reste pas à l'écart des événements du monde. L'espace méditerranéen nord-africain, éminemment multiculturel, a connu des soulèvements appelés communément le « Printemps arabe ». Nous nous intéressons à l'influence de cet évènement sur la communication touristique et nous cherchons à identifier les stratégies de communication adoptées par la Tunisie

et l'Égypte, pays de forte tradition touristique, pour s'adapter aux évolutions géopolitiques et à leurs répercussions. Comment en période de post-crise, lorsqu'une

forme de stabilité est rétablie, les institutions touristiques s'adressent-elles aux visiteurs et particulièrement à leur clientèle francophone pour les motiver à revenir?

Nous supposons que l'évolution de la promotion touristique intervient au niveau des genres de discours, responsables des stratégies argumentatives. Afin de répondre à nos

interrogations, $\mathrm{n}$

ous avons collecté des supports de communication multimédiatiques, émis par les autorités des deux pays après les événements révolutionnaires de 2011 et jusqu'en 2015 ${ }^{1}$ et, $n$

ous avons élaboré une grille d'analyse à deux volets (linguistique et visuel) pour déboucher sur une interprétation globale des documents en contexte. 
Au départ, notre corpus est construit

à partir de deux ressources institutionnelles : physiques comme les bureaux culturels de l'Égypte et de la Tunisie en France ; virtuelles comme les sites Internet des offices de tourisme et leurs pages Facebook. Nous avons ajouté des ressources virtuelles non professionnelles en tourisme qui évoquent l'actualité touristique.

Afin de cerner l'éventuel impact de la crise sur le discours promotionnel touristique, nous nous référons à des documents touristiques émanant de différents genres, supports et sources, qui nous semblent aptes à jouer un rôle marquant dans la promotion touristique. Nous étudierons ici un échantillon de sept iconotextes

de ce corpus : livret promotionnel du bureau culturel de l'ambassade de l'Égypte (1), page Facebook officielle de promotion touristique en Égypte (2), campagne électronique tunisienne (3), newsletter

électronique de promotion officielle du tourisme tunisien (4), tweet français de soutien à la Tunisie (5), chronique littéraire tunisienne publiée dans un magazine féminin (6), article électronique d'actualité maghrébine (7).

En effet notre objectif exige d'abord, pour appréhender la richesse culturelle et argumentative de nos documents, de les situer au sein des genres de discours touristiques habituels d'appartenance avant d'aborder les genres inhabituels.

Pour rendre compte de la complexité socio-discursive de ce corpus, nous avons adopté une base théorique plurielle comme y invite d'ailleurs notre discipline principale,

l'analyse du discours (Maingueneau, 2004 ; Rabatel, 2016) : nous recourons à la psychologie sociale (Deschamps \& Moliner, 2008), la linguistique textuelle (Adam, 2011), la sémiotique visuelle appliquée ( Fozza

et

al.,

, l'argumentation sous l'angle général de la publicité (Adam \& Bonhomme, 2010), et plus spécifiquement de la promotion touristique qui nous apparaît modulée selon des genres variés du discours, associant propriétés formelles, inscription dans les contextes Mourlhon-Dallies, 2000).

et visées pragmatiques (

Nous nous demanderons donc comment les iconotextes apparentés à la promotion touristique pratiquent une valorisation appropriée au contexte méditerranéen de postcrise, en faisant appel aux ressources de l'argumentation, à travers la variété des genres du discours et des mises en scène visuelles. Nous faisons plusieurs hypothèses dans le prolongement de cette problématique.

Tout d'abord, les professionnels du tourisme s'appuient sur une base culturelle de représentations sociales à fonction argumentative adressées au public occidental, qui se rattache à l'imagologie ${ }^{3}$ et à son jeu de miroirs, puisqu'il s'agit d'une « analyse du contenu des représentations qu'un peuple se fait d'un autre, (hétéro-images) et de luimême (auto-image) »(Amossy \& Herschberg-Pierrot, 2005: 46).Nous entendons par argumentation l'ensemble des techniques rhétoriques pour agir par la parole en s'appuyant sur des points d'accord et les évidences partagées par l'auditoire (Perelman cité par Amossy, 2014 : 112).

7 Ensuite, la communication touristique, ne s'appuie pas que sur les genres ouvertement promotionnels, mais aussi sur des genres liés à l'actualité politique, littéraire, économique qui apportent un soutien au discours touristique de manière indirecte, du 
fait des caractéristiques techniques et sémiotiques provenant des supports médiatiques. Cette reconfiguration de genres touristiques s'accorde avec l'hybridité que leur reconnait Kerbrat-Orecchioni à propos des guides touristiques (2004: 134). L'intérêt est d'internaliser les informations pour le lecteur (Décaudin \& Bouguerra, 2011) c'est-à-dire, de transmettre un message dans le système de valeurs de son récepteur. Les professionnels recourent ainsi à l'aspect pseudologique ${ }^{4}$ de l'argumentation en donnant l'illusion de faire plus que de communiquer une information (Freudiger, 1988).

Enfin, l'argumentation touristique directe ou indirecte est de nature plurisémiotique, et le volet visuel est aussi important que le volet linguistique (Dondero, 2014), pour solliciter le sensible sur le plan de la perception et de l'imaginaire. Nous illustrerons cet aspect notamment à travers l'identification du monde de référence des éléments iconiques et les techniques photographiques qui valorisent ces derniers. Les éléments iconiques et plastiques, comme les éléments linguistiques, remplissent, dans les documents touristiques, un rôle argumentatif à soubassement culturel et identitaire permettant de susciter des émotions et des souvenirs (Azaïz, 2017).

Dans un premier temps nous étudierons la reconfiguration des genres et dans un deuxième temps les stratégies argumentatives plurisémiotiques.

\section{Une reconfiguration des genres du discours touristique : de la promotion au soutien}

10 Plus que la définition littéraire des genres (prose et poésie, théâtre, roman, essai, etc.), la distinction aristotélicienne des genres rhétoriques (épidictique, délibératif, judiciaire) associe les formes d'expression aux pratiques sociales. C'est dans cette tradition, à partir de la réflexion initiée par Bakhtine, qui conçoit le genre comme un espace de régularités et de contraintes «entre le système collectif et arbitraire de la langue et l'“aléatoire" de la parole»(Richer, 2011), que les genres de discours se particularisent et se diversifient, en phase avec le développement social. Nous reprenons la définition des genres de discours comme «des institutions de parole articulant une identité énonciative avec un lieu social ou avec une communauté de locuteurs» (Charaudeau \& Maingueneau, 2002: 281). Il s'agit de configurations discursives relativement malléables, issues d'une pratique sociale, comportant des traits textuels, pragmatiques et sémiotiques. Le genre module l'impact de l'argumentation sur un public cible en fonction de ses caractéristiques et des objectifs pragmatiques de départ.

11 Afin de caractériser de manière opératoire les genres des iconotextes retenus ici pour notre analyse $e^{5}$ nous nous référons aux traits définitoires des genres de discours proposés par Richer (2011) selon cinq dimensions : thématique, matérielle (c'est-à-dire relative au support), textuelle, socio-pragmatique (c'est-à-dire relative aux énonciateurs et aux actes de discours), stylistique. Pour ce qui est de la dimension thématique, tous les documents de notre corpus se rattachent au tourisme ${ }^{6}$ et leurs supports matériels sont soit papier (1), soit électroniques $(3,4,5,6,7)$, soit les deux (2, 6). Ce sont les dimensions textuelle, socio-pragmatique et stylistique qui les différencient.

12 Nous pouvons diviser nos iconotextes en deux groupes. Le premier relève d'une stratégie de promotion directe à travers des genres préalablement consacrés à la 
promotion touristique (le livret $-1-$, les campagnes multi-supports -2 et $3-$, l'éditorial -4 ). Les énoncés du deuxième groupe (le tweet $-5-$, la chronique $-6-$, l'article -7) n'émanent pas directement des instances de l'industrie du tourisme mais favorisent celle-ci en relayant des échos positifs et en lui apportant un soutien. Les tableaux ci-dessous présentent chaque document avec des informations factuelles (nom, source, support et date), ainsi que la cible visée, une caractérisation du genre et du type d'argumentation, plutôt rationnelle ou plutôt émotionnelle.

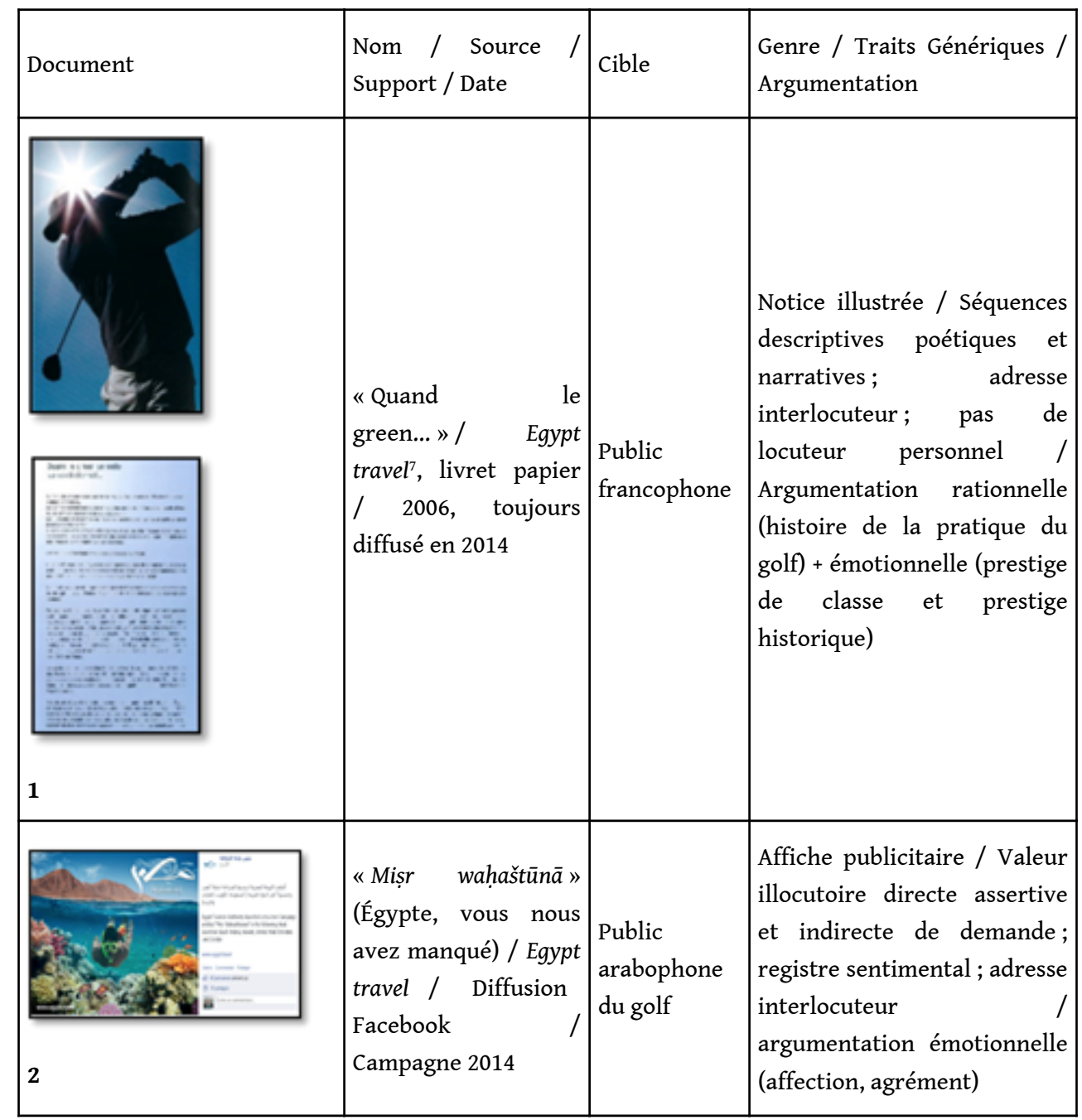




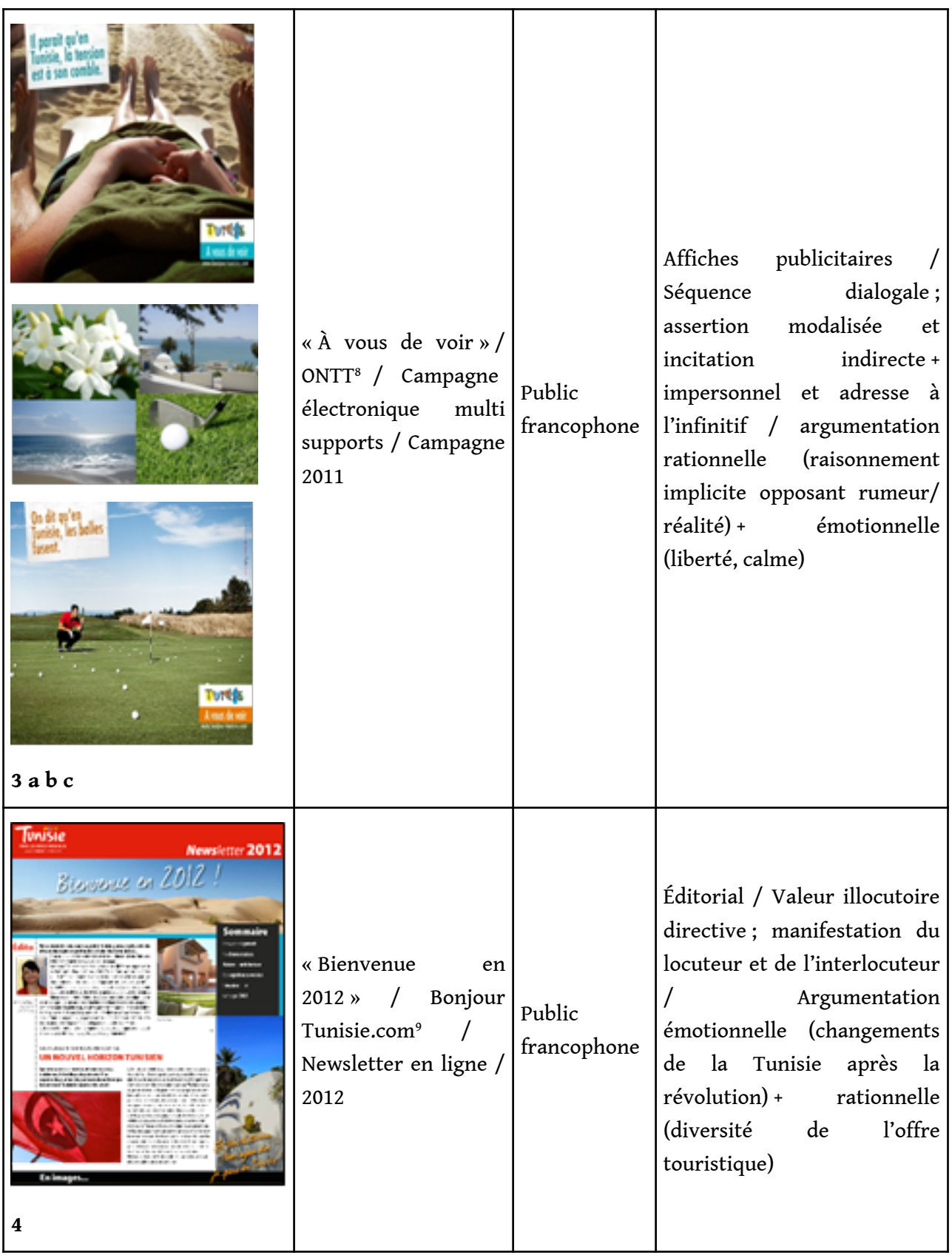

Tableau 1. - Documents de promotion directe.

13 Nous remarquons que toutes les instances émettrices de ce groupe (tableau 1 supra) sont des instances touristiques qui font une présentation idéale de la destination à travers les genres « promotionnels classiques » de la notice, de l'affiche de campagne et de l'éditorial d'une newsletter, et que les documents tunisiens intègrent le contexte post-crise.

\begin{tabular}{|l|l|l|l|}
\hline Document & $\begin{array}{l}\text { Nom / Source / } \\
\text { Support / Date }\end{array}$ & Cible & $\begin{array}{l}\text { Genre / } \\
\text { Traits } \\
\text { génériques / } \\
\text { Argumentation }\end{array}$ \\
\hline
\end{tabular}




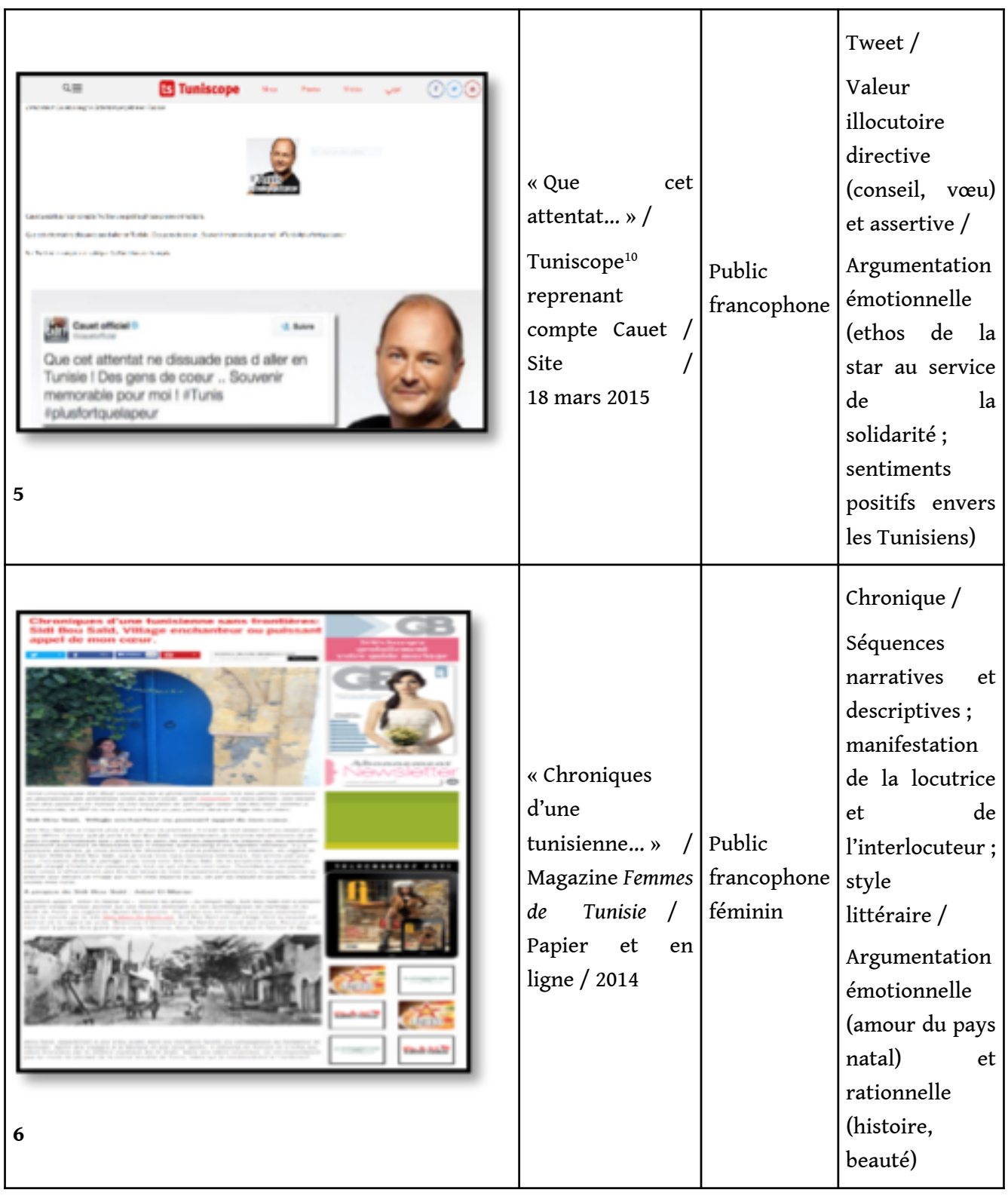




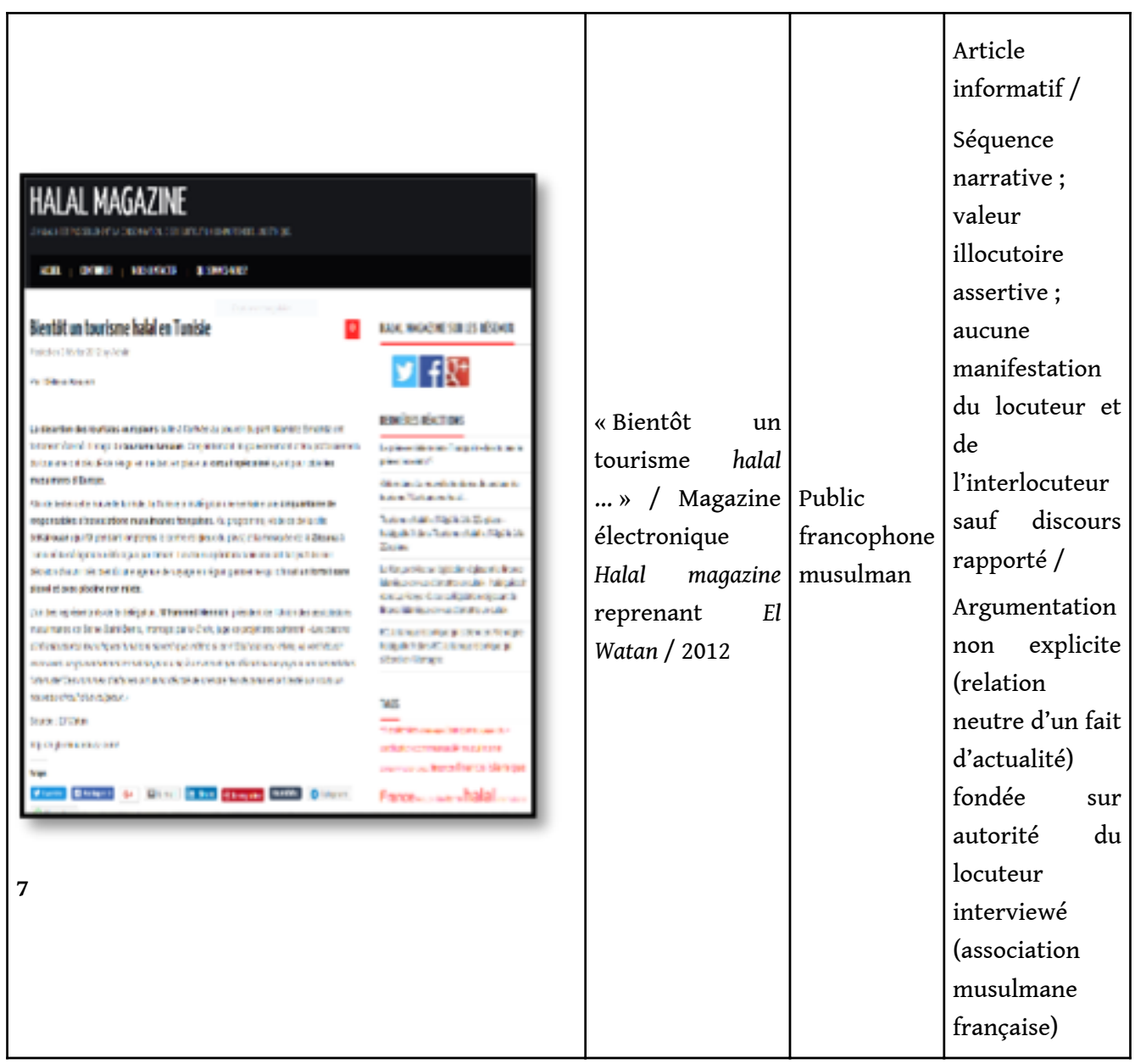

Tableau 2. - Documents de promotion indirecte.

14 Le deuxième groupe (tableau 2 supra) rassemble des documents collectés grâce à notre veille des pages Facebook des autorités touristiques tunisiennes qui guettaient les attitudes positives envers le tourisme tunisien. En effet, Facebook a connu un saut fonctionnel dès le début du Printemps arabe car il a réussi à être une plateforme d'échange d'informations, de vidéos et d'images dans le monde arabe afin de suivre les actualités censurées dans les médias officiels. L'arrivée de la démocratie en Tunisie à cette époque a fait que les instances officielles du tourisme utilisent ce réseau social afin de diffuser l'actualité touristique notamment auprès des populations jeunes. Par contre, la stratégie de communication touristique officielle de l'Égypte, restée méfiante et plus restrictive quant à ce type d'utilisation, n'a pas permis de recueillir de documents de promotion indirecte, comme nous l'avons fait pour la Tunisie.

Les documents du deuxième groupe ont pour point commun de soutenir la Tunisie et son tourisme sans se mêler ouvertement de promotion professionnelle, d'où vient notre dénomination de "promotion indirecte ». Ils le font à travers les genres du billet de réseau social, de la chronique journalistique et littéraire, de l'article informatif. Ces énoncés s'adressent à des cibles différentes avec des arguments différents (solidarité, agrément, religion). 
16 La communication touristique dispose donc, suite à la recherche de renforts médiatiques pendant la crise, de genres malléables qui sont susceptibles d'intégrer et de recycler des genres établis pour d'autres usages sociaux et de se renouveler ainsi.

Comment les discours des genres promotionnels et non promotionnels parviennent-ils à valoriser les destinations et à persuader de potentiels visiteurs? Afin de déterminer la relation entre genres discursifs et argumentation, nous avons privilégié des entrées d'analyse textuelle et pragmatique d'une part, d'analyse icono-plastique (dans le sillage de Barthes, 1964) d'autre part.

\section{Genres de discours et stratégies argumentatives}

Pour le volet linguistique des documents, nous nous sommes surtout intéressé à deux dimensions inspirées du modèle générique de Richer (2011) : la dimension textuelle qui concerne la dominante séquentielle, ainsi que la dimension socio-pragmatique qui porte sur l'inscription des protagonistes de l'énonciation (locuteur et son interlocuteur) dans le texte et sur les actes de discours, dans la mesure où «[...] un texte est défini comme une séquence d'actes de discours qui peut être considérée ellemême comme un acte de discours unifié » (Adam, 2011 : 36).

En examinant la dimension textuelle des différents documents, nous avons constaté que la séquence descriptive est dominante pour les documents $1,4,6$, grâce à sa capacité de faire imaginer ce que le lecteur ne connait pas. Pour les documents 2 et 3 , en l'absence de description verbale, l'image y est suppléée alors que, pour les documents 1,4 et 6 , elle complète la description. La description porte sur les avantages du pays en abordant des mythes liés à l'histoire de celui-ci ; ce qui donne une modalité appréciative positive de cette description et exerce donc un rôle argumentatif dans le livret (1), l'éditorial (4) ou encore quand la chroniqueuse décrit poétiquement son village natal (6).

Les séquences à dominante narrative se trouvent aussi bien dans la notice sur le golf avec un rappel historique (1), que dans la chronique où l'auteure retrace l'histoire de la ville et son propre itinéraire (6), ou que dans l'article de Halal magazine qui évoque l'actualité politique (7), ou encore dans l'éditorial qui recourt à une sorte d'aphorisme poétique pour évoquer les événements "quand le vent de la liberté souffle dans les urnes tunisiennes, il n'oublie pas la palmeraie » (4). Cependant les registres stylistiques dans ces supports sont très différents, allant du neutre (7) au lyrique $(1,4,6)$. La dominante narrative de ces séquences a pour fonction d'atteindre l'imaginaire en passant par la réalité (Freudiger, 1988: 224). Le récit donne une base factuelle à l'argumentation, mais il peut se conjuguer à l'émotion. La dimension textuelle n'est donc pas discriminante pour ce qui est de la promotion directe ou indirecte, car les options se trouvent dans les deux groupes de documents.

Pour cerner la dimension sociopragmatique, nous nous centrons surtout sur les pronoms personnels et les actes de langage (Searle, 1972), appelés également actes de discours. L'appel aux émotions ou au pathos qui relève de la dimension pragmatique, est présente dans les deux groupes de documents, mais, dans le deuxième, les émotions renvoient à une expérience et à des sentiments personnels, comme l'atteste l'emploi de la première personne référant à une locutrice («mon Sidi Bou Saïd», 6) ou un locuteur (« des gens de cœur... souvenirs mémorables pour moi », 5), bien identifiés. Dans les 
genres directement promotionnels, ce sont des clichés lyriques qui semblent porter l'argumentation, en recourant aux expressions convenues, mais sans implication vécue: «Les palmiers se balancent doucement au rythme de la brise marine rafraichissante et leurs feuilles semblent caresser le ciel d'un bleu profond »(1), tout comme l'éloge plus politique de l'éditorial «plus responsable, plus haut de gamme et plus authentique, le tourisme en Tunisie fait aussi sa révolution » (4).

Si nous comparons les deux documents dont le contenu est le plus général $(4,7)$, nous constatons que la majorité des actes de discours sont assertifs pour présenter des informations au lecteur. Mais les finalités diffèrent car le magazine (7) énonce des éléments objectifs ( une agence de voyage qui offrirait un forfait sans alcool avec piscine non mixte») sans chercher apparemment à convaincre le lecteur, mais en s'appuyant sur la force de données présentées comme factuelles et sur des valeurs partagées, alors que l'éditorial promotionnel (4) explicite les valeurs avec un engagement beaucoup plus fort et une adresse aux lecteurs; les actes assertifs de l'éditorial ont une valeur dérivée nettement directive. C'est une autre sorte d'engagement qui intervient dans la chronique : elle comporte des actes assertifs et expressifs avec une forte présence du pronom « je » de la narratrice amoureuse de son village «Cet article est pour moi, l'occasion rêvée de partager avec vous mon Sidi Bou Saïd $»(6)$.

23 L'acte de discours assertif peut se charger de plusieurs valeurs illocutoires, comme dans le cas du slogan («Mișr : wahaštūnā », c'est-à-dire « Égypte : vous nous avez manqué ») qui a une valeur d'expressif mais aussi de directif. Le locuteur s'adresse à sa cible comme à une personne proche. Toutefois, si l'interlocuteur est interpellé, le locuteur n'a pas de consistance. L'acte directif peut passer par un impératif «Vous aussi, découvrez les trésors du golf... » (1) ; c'est le cas également dans l'éditorial (4) où, dès l'accroche, la locutrice adresse trois actes de discours directifs incitatifs à son interlocuteur, signes d'une volonté de rapprochement avec la cible pour préparer un climat de connivence : "Venez découvrir le nouveau visage de la Tunisie, goûtez à la diversité des offres et des régions et profitez de la chaleur des terres du Sud... ». Mais contrairement au document égyptien (1), la locutrice de l'éditorial (4) est présente et davantage incarnée par le pronom «nous» qui l'intègre («Nous vous invitions à découvrir ce pays avec un autre regard ») et par la signature ${ }^{11}$. En revanche l'article de Halal Magazine (7), écrit à la troisième personne, ne comportant que des actes assertifs, s'abstient de toute inscription du locuteur et de toute adresse explicite à un lecteur, en reprenant des informations et un discours rapporté : c'est indirectement, de par sa propre ligne éditoriale, qu'il argumente pour un tourisme "halal », c'est-à-dire en conformité avec les préceptes religieux, en accord avec le gouvernement tunisien de l'époque. L'article n'est accompagné d'aucun visuel, mais la sobriété de la présentation et de la typographie s'accorde bien avec un magazine qui porte sur des sujets concernant les pratiquants musulmans dans une optique conservatrice.

Si la plupart des documents (1 à 6) mettent en scène un simulacre de dialogue en s'adressant à un public, dans la campagne tunisienne de 2011 (document 3), les actes de discours assertifs s'intègrent à une séquence dialogale globale qui a une valeur directive. Les assertions «Il paraît qu'en Tunisie, la tension est à son comble », « On dit qu'en Tunisie, les balles fusent » pourraient venir d'un touriste qui rapporte des propos anonymes négatifs. Mais la réfutation de ces assertions venant des instances tunisiennes ("à vous de voir») passe non par la description, mais par l'image qui 
montre le contraire, nous y reviendrons. Cette campagne promotionnelle ludique et audacieuse repousse les on-dit et valorise le public, car elle le rend partenaire en le faisant réfléchir au flot des assertions trompeuses pour l'inviter à ne pas les croire, mais à juger par lui-même. La valorisation, passant par la connivence avec l'interlocuteur, est essentielle dans les documents promotionnels censés faire accepter une proposition à l'interlocuteur.

Cette volonté est manifestée aussi dans l'éditorial grâce à l'appel à la visite de la Tunisie (4). Les deux documents ( 3 et 4 ), s'ils relèvent de la promotion directe et de genres touristiques traditionnels, s'adaptent cependant au contexte géopolitique en renvoyant allusivement ou directement à la situation, et en sollicitant la participation active du récepteur. Ils restent toutefois dans le cadre de l'éloge d'activités et de sites. Dans le billet de tweet (5) en revanche, on constate à la fois l'engagement personnel d'une célébrité ${ }^{12}$ et l'implication de l'interlocuteur appelé au soutien de la Tunisie par l'expression au subjonctif («Que l'attentat ne dissuade pas d'aller en Tunisie »).

L'argumentation fait le plus souvent appel à la fois au raisonnement et à l'émotion en impliquant la cible en tant qu'interlocuteur, mais la palette des émotions varie selon les pays et l'adaptation ou non aux situations post-crise.

Une ressource argumentative majeure des genres du discours touristique est la valorisation par les visuels : les éléments icono-plastiques relayant le lexique ou étant relayés par ce dernier partent d'une donnée perceptive pour la charger de connotations séduisantes. On pourrait parler d'une dimension stylistique plurisémiotique. Comme le remarque Kerbrat-Orecchioni à propos de prospectus : «La valorisation concerne [...] différents thèmes [...] : les paysages naturels, le patrimoine historique et culturel, le climat, et les habitants [...]» $(2004: 138)$.

La part visuelle est productrice du sens en relation harmonieuse ou éventuellement conflictuelle avec le sens produit par le texte: elle l'affirme ou le complète. Elle participe à la construction du genre de discours. Notre recherche aborde cette composante sous le biais des éléments iconiques représentés et de certains éléments plastiques comme les techniques de prise de vue photographiques ou les éléments chromatiques et leurs connotations.

On constate l'alliance du naturel et du culturel qui convergent pour exalter un sport dans le livret égyptien (1). En effet, dans le titre "Quand le green se mêle au soleil éternel » (1) le « soleil éternel » que l'on retrouve éblouissant dans l'image ne concerne pas seulement le climat, mais il connote l'emblème divin des Pharaons. Les bras du joueur, photographié en pleine action avec une légère contre-plongée, sont saisis dans un mouvement qui dessine une pyramide, rappelant le trésor pharaonique de l'Égypte, une auréole de soleil figurant à côté de la tête du joueur. Le "green $»^{13}$ n'apparaît pas sur la photo. Ce terme anglais suggérant la distinction sociale désigne le terrain de golf à travers la métonymie de la couleur verte de la pelouse. Aux côtés d'autres mots spécialisés (" fairways, links »), il témoigne de la mondialisation amenant l'introduction dans un texte francophone de mots anglais pour s'adresser à une clientèle aisée. D'autres mots mélioratifs ou soutenus concernant la couleur (« les fairways verdoyants ondoient comme des tapis volants couleur d'émeraude ", «l'azur inimitable ») insistent sur la beauté et l'agrément en évoquant des matières précieuses et un univers de contes orientaux ("tapis volants ${ }^{14}$ ). Cela confirme l'aspect classique de la promotion touristique de l'Égypte qui s'appuie sur son patrimoine historique même dans un livret consacré au sport. Le concepteur n'hésite pas à mobiliser les stéréotypes culturels 
quand il met en relief la richesse architecturale des pyramides à côté du rappel de l'histoire du golf. Mourlhon-Dallies comparant le discours touristique consacré à différents pays méditerranéens souligne que les publicités pour l'Égypte vantent les vestiges tout en mettant en avant un voyage à remonter le temps et une séparation entre présent et passé $(2000: 129)$.

Les mises en scène des éléments iconiques, dans les photos de la campagne tunisienne de tourisme consacrées au golf ( $3 \mathrm{~b}$ et $\mathrm{c}$ ), suggèrent le calme et l'harmonie avec un gros plan sur la canne et la balle blanche (blanche comme le jasmin et les habitations), un paysage maritime ensoleillé ; un joueur en tenue sur un terrain de golf vu en plan d'ensemble. Mais au lieu de mettre en avant la richesse historique comme l'Égypte, cette campagne tunisienne de 2011 (3) n'élude pas la crise, et fait face aux rumeurs qui assimilent la Tunisie à un pays en guerre. Le texte ne réfère pas à l'histoire de la Tunisie, mais exploite la polysémie lexicale, tandis que le visuel focalise sur de nombreuses balles de golf. Celles-ci sont au premier plan par rapport au reste du paysage naturel verdoyant avec une montagne en arrière-plan grâce à la grande profondeur du plan d'ensemble de la photo. La mise en scène de ce paysage signifie la densité de la pratique du golf en Tunisie par les touristes tout en jouant sur la polysémie de "balles » (armes / sport), "On dit qu'en Tunisie, les balles fusent », afin de réfuter la rumeur alarmante par l'image paisible. Le genre du discours est directement promotionnel mais recourt à un conflit image / texte et à l'ironie pour assumer la post-crise.

31 L'idéalisation d'un paysage emblématique est entièrement portée par l'image pour l'affiche d'Egypt travel reprise sur Facebook (2). Selon Damien, « la contemplation d'un paysage emblématique constitue le moment réussi, heureux du voyage : cette vision cristallise dans un éblouissement les attentes et les espoirs du touriste. C'est elle qui à la fois motive le déplacement, le récompense et lui donne son sens.» (2003: 16) La focalisation photographique sur certains éléments visuels pour exalter le charme des vacances est une donnée commune aux documents touristiques de tous les pays du monde.

Ainsi

un paysage réunissant des montagnes, le ciel clair et la mer calme renvoie à un aspect récurrent dans la promotion touristique. Tous ces éléments iconiques sont présents dans l'affiche (2) où

le bleu du ciel répond au turquoise de la mer; le plongeur est au centre de l'image avec à l'arrière-plan les montagnes arides et au premier plan la flore et la faune sous-marine multicolore. Le paysage est méditerranéen, sans ancrage spatio-temporel très précis, et la densité des éléments ainsi que l'harmonie des couleurs encouragent la découverte de la nature du pays dont le nom est donné par le slogan. Dans les documents 1 et 3 également, le bleu domine avec sa valeur usuelle de «beau temps » exaltant la nature et convenant particulièrement au tourisme balnéaire. La récurrence du bleu s'explique par le fait que cette couleur est la plus immatérielle. Grâce à elle, le regard s'enfonce sans rencontrer d'obstacles pour se perdre dans l'infini (Chevalier \& Gheerbrant, 1997 : 129). Les couleurs ne font pas sens universellement mais en vertu d'effets connotatifs pour construire ce que les spécialistes de marketing appellent un «ADN de marque » (Corbille, 2013).

32 Les couleurs peuvent se transformer en une marque de particularité si la couleur est rattachée à d'autres éléments ancrés dans le terrain culturel. Ainsi la couleur bleue des portes, valorisée par la prise photographique en plan de semi-ensemble, contrastant 
avec le blanc (document 6) est liée spécifiquement à l'architecture traditionnelle de la Tunisie $^{15}$. Dans ce cas la particularité plastique représente le fait culturel. Le texte de la chronique (6) s'inscrit tout à fait dans la tradition d'idéalisation touristique comme le montrent ces passages qui associent nuance chromatique et jugement positif à propos d'une localité très connue et d'un élément typique : «Derrière chaque porte au bleu féerique, se cache un monde nouveau "; "Tunisiens et Tunisiennes, sachez que l'on doit au baron [le baron d'Erlanger] la signature de notre village qui en fait sa renommée mondiale, ce bleu et blanc féerique et majestueux que l'on affectionne tant. » Les adjectifs «féerique », «majestueux» insistent sur l'exception. Les photographies qui accompagnent la chronique relèvent de prises de vue réalistes et personnalisées. En effet, on voit deux fois la jeune femme devant une des fameuses portes. L'originalité de la chronique tient à la subjectivité émotionnelle et personnelle admise dans le discours littéraire, et que le texte tout comme l'image traduisent bien.

Les paysages emblématiques naturels ou urbains connotent un cadre oriental (sable $-2,3,4-$, jasmin $-3-$, demeures tunisiennes -3 et $6-$ ), mais nos documents intègrent aussi des éléments « occidentaux » tels que les cannes et les balles de golf ( 1 et 3) ou sur le plan des mœurs, dans le document 3a, le couple sur le sable. En effet la position allongée décomplexée (l'un couché sur l'autre et les pieds en éventail) évoque les trois $S$ (Sea, sun and sex) ${ }^{16}$ et la tranquillité d'un couple qui se prend en selfie. Cette campagne tunisienne met ces éléments visuels au service du rapprochement basé sur la connivence avec le monde occidental en mettant en relief la liberté de comportement en Tunisie. L'argumentation implicite est de rassurer sur la sécurité (3c) comme sur la liberté (3a), en montrant des images assez banales qui contredisent les messages alarmistes. "Non la tension n'est pas à son comble puisqu'on peut faire cela » indique l'image qui assure une fonction de relais particulier ${ }^{17}$. Le verbe «voir» («à vous de voir ») renvoie au visuel et aussi et surtout à la situation.

En cherchant toujours l'adaptation de l'argumentation à la post-crise, nous retrouvons les couleurs des paysages méditerranéens dans les photographies qui accompagnent l'éditorial (4) : ocre du sable, bleu du ciel et de la mer, blanc des habitations. Mais nous remarquons aussi la présence inhabituelle du rouge qui est celle du bandeau du site, du vêtement visible de la locutrice, du drapeau tunisien, dont on trouve d'ailleurs une photo plus bas, sans oublier la connotation révolutionnaire de cette couleur. Or cet éditorial fait directement la promotion de la Tunisie mais en affirmant l'évolution positive du tourisme due au changement politique. Le genre de l'éditorial touristique a donc subi, de l'intérieur du genre, une transformation suite à la crise. C'est ce qui autorise la présence inhabituelle d'un symbole iconique politique dans un document touristique pour affirmer la réussite de la révolution tunisienne, et son impact positif à la fois sur la cohésion nationale tunisienne et sur le tourisme.

Au terme de cette analyse plurisémiotique, nous pouvons dire que les traits génériques textuels et pragmatiques se combinent avec les traits stylistiques visuels sans qu'il soit possible d'établir une correspondance simpliste entre tel genre et telle stratégie argumentative. L'adaptation au contexte passe certes par le renouvellement des genres mais aussi par la mobilisation de certaines représentations. 


\section{Conclusion}

À partir d'un corpus multi-médiatique dans lequel texte et image prennent part à la construction du sens, nous avons observé que la communication touristique se caractérise par une certaine malléabilité, adoptant des genres variés qui peuvent en intégrer d'autres et aller de la promotion au soutien. Nous avons constaté qu'à travers leurs iconotextes, les genres de discours de promotion ou de soutien touristique mobilisent chez leurs destinataires des connaissances préalables et des représentations de l'autre, en cherchant à les conforter ou à les modifier.

Les autorités du tourisme tunisien font de l'adaptation au nouveau contexte une priorité qui n'existe pas dans notre corpus égyptien, classique dans ses représentations. D'un côté, la crise du tourisme est évoquée, et l'argumentation en faveur d'un séjour en Tunisie et des activités pouvant y être pratiquées en tient compte, procédant souvent de manière indirecte à partir de séquences descriptives qui prennent une valeur incitative. D'un autre côté, les techniques de mises en scène du visuel ont mis en valeur certains éléments. Cette alliance du discursif et du visuel produit une image qui conjugue tradition et modernité, construit une harmonie naturelle et culturelle et propose des expériences intenses.

\section{BIBLIOGRAPHIE}

\section{Corpus}

BADJI Atif (2014), « Chronique d'une tunisienne sans frontières », en ligne sur Femmes de tunisie.com : <http://femmesdetunisie.com/> (10 mars 2015).

Krazim Mélissa (2012), «Bientôt un tourisme halal en Tunisie », en ligne sur Halal Magazine : <http://www.halalmagazine.com/bientot-un-tourisme-halal-en-tunisie/> (3 février 2012).

OFFICE DE TOURISME ÉGYPTIEN (2014), « Misr wahachtouna », en ligne sur Misr bedayat al hekayah-

Experience egypt : <https://www.facebook.com/pg/fr.experienceegypt/photos/?ref=page internal> (26 mai 2014).

OfFICE DE TOURISME ÉGYPTIEN (2014), « Quand le green se mêle au soleil éternel... », Greens intenses sous un soleil d'or..., Paris : Dumoulin.

OFFICE DE TOURISME TUNISIEN (2011), Campagne publicitaire pour la relance du tourisme tunisien, Lyon : Memac Label Tunisie.

OfFICE DE TOURISME TUNISIEN (2012), « Édito », en ligne sur Newsletter 2012 : <www.bonjourtunisie.com> $\left(1^{\mathrm{er}}\right.$ janvier 2014).

TUNISCOPE (2015), « Que cet attentat ne dissuade pas d'aller en Tunisie », en ligne sur Cauet : que cet attentat ne dissuade pas d'aller en Tunisie : <http://tuniscope.com/article/65274/tech/social/ cauet-063711> (18 mars 2015). 


\section{Ouvrages et articles}

ADAM Jean-Michel (2011), Les textes : types et prototypes ( $3^{\mathrm{e}}$ éd.), Paris : Armand Colin.

ADAMJean-Michel \& BonHommeMarc (2010),

L'argumentation publicitaire : rhétorique de l'éloge et de la persuasion[1997], Paris : Armand Colin.

Amossy Ruth (2014), L'argumentation dans le discours [2012], Paris : Armand Colin.

AMOSsY Ruth \& HERSCHBERG-PIERROT Anne (2005), Stéréotypes et clichés, Paris : Armand Colin.

AZAïz Hanna (2017), « La signification des couleurs pour influencer votre marketing », en ligne sur 1 min30 : <https://www.1min30.com/identite-visuelle/signification-couleurs-influencermarketing-124725\#0> (9 juillet 2018).

BARTHES Roland (1964), « Rhétorique de l'image », Communication (4), 40-51, < https://www.persee.fr/doc/comm_0588-8018_1964 num_4_1_1027> (10 décembre 2018).

ChaRAUdeau Patrick \& Maingueneau Dominique [dir.] (2002), Dictionnaire d'analyse du discours, Paris : Seuil.

CHEVAliER Jean \& GHEERBRANT Alain (1997), Dictionnaire des symboles [1969], G. Schoeller (dir.), Paris : Éditions Robert Laffont \& Éditions Jupiter.

CORBILLE Sophie (2013), « Les marques territoriales : Objets précieux de l'économie de la renommée ", Communication, 32(2), <http://communication.revues.org/5014> (12 mai 2014).

DAMIEN Elsa (2003), « L'Italie touristique du XIX ${ }^{\mathrm{e}}$ siècle : la fixation de quelques paysages emblématiques ", Chroniques italiennes (4), <http://chroniquesitaliennes.univ-paris3.fr/PDF/ Web4/Damien03.pdf> (6 décembre 2018).

DECAUdIN Jean-Marc \& BOUGUERRA Afifa (2011), La communication marketing intégrée, Paris : Économica.

DONDERO Maria Giulia (2014), « Photographier le travail, représenter le futur. Iconographie du chantier des Halles à Paris », Communication \& langages (180), 79-93, <https://www.cairn.info/ revue-communication-et-langages1-2014-2-page-79.htm> (24 janvier 2019).

FozzA Jean-Claude et al. (1988), Petite fabrique de l'image : parcours théorique et thématique, Paris : Magnard.

FREUDIGER Marc-André (1988), « Narration et communication indirecte à partir de Kierkegaard », P. Bühler \& J.-F. Habermacher (dir.), La narration : quand le récit devient communication, Genève : Labor et Fides, 217-229.

KERBRAT-ORECCHIONI Catherine (2004), « Suivez le guide ! Les modalités de l'invitation au voyage dans les guides touristiques : l'exemple de l'île d'Aphrodite », F. Baider et al. (dir.), La communication touristique : approches discursives de l'identité et de l'altérité, Paris : L'Harmattan, 133-150.

MAINGUENEAU Dominique (2004), « Retour sur une catégorie : le genre », J.-M. Adam et al. (dir.), Texte et discours : catégories pour l'analyse, Dijon : Éditions universitaires de Dijon, 107-118.

MOURLHON-DAllies Florence (2000), « Les prospectus d'office de tourisme sur l'Italie du Nord: introduction à la notion de "particularisme discursif" ", M. Margarito (dir.), L'Italie en stéréotypes : analyse de textes touristiques, Paris : L'Harmattan, 127-152. 
NERLICH Michael (1990), «Qu'est-ce qu'un iconotexte ? Réflexion sur le rapport texte-image photographique dans La femme se découvre », A. Montandon (dir.), Iconotextes, Paris : Ophrys, 255-302.

RABATEL Alain (2016), « Postures énonciatives, variable générique et stratégies de positionnement », J. Angermuller \& G. Philippe (dir.), Analyse du discours et dispositifs d'énonciation : autour des travaux de Dominique Maingueneau (recueil et présentation), Limoges : Lambert-Lucas, 125-135.

RICHER Jean-Jacques (2011), « Les genres de discours : une autre approche possible de la sélection de contenus grammaticaux pour l'enseignement / apprentissage du FLE ? », Linx (64-65),

<https://journals.openedition.org/linx/1396> (9 juillet 2018).

SEARLE John (1972), Les actes de langage, Paris : Hermann.

\section{NOTES}

1. La limite de 2015 correspond à une certaine stabilité politique pour la Tunisie (transition démocratique, adoption de la Constitution début 2014, élections législatives et présidentielles fin 2014) comme pour l'Égypte (élections présidentielles de 2014, législatives de 2015, régime autoritaire du président Al-Sissi).

2. Le concept d'iconotexte trouve son origine dans la pratique artistique et photographique pour traiter le rapport dialectique de l'illustratif de l'image à la narration

(Nerlich, 1990 : 259). Il est repris notamment par Adam (2011) et Adam \& Bonhomme (2010) pour désigner des productions mixtes (verbales et visuelles) dans le domaine de la presse ou de la publicité en particulier. C'est pourquoi nous le reprenons ici pour désigner des documents sémiotiquement mixtes. Les références des documents du corpus figurent en bibliographie. 3. Le contenu des représentations qu'un peuple se fait d'un autre (hétéro-images) et de lui-même (auto-image).

4. Cet aspect désigne la fausse apparence de l'argumentation qui dissimule la finalité marketing par une finalité relationnelle feignant de favoriser les intérêts du lecteur potentiel.

5. Ils figurent ci-dessous dans deux tableaux récapitulatifs et intégralement en annexe.

6. C'est-à-dire selon Meyer (2005) l'ensemble des phénomènes liés au déplacement hors des lieux de résidence en vue de l'agrément et des loisirs.

7. Il s'agit de l'autorité égyptienne du tourisme.

8. Office National de Tourisme Tunisien.

9. Site professionnel. L'éditorial émane de la directrice de l'ONTT.

10. Site professionnel.

11. Mme Hachani, la directrice de l'ONTT en France.

12. Ainsi le seul visuel du tweet est la photo choisie par son auteur, comme c'est l'usage.

13. Ce terme désigne la surface gazonnée et tondue ras sur laquelle se trouvent les trous.

14. Nous pensons également à une nuance mystique du vert qui indique «renouveau, résurrection - vêtements des habitats du Paradis [...] (Chevalier \& Gheerbrant, 1997 : 923).

15. Trois des photos qui accompagnent le texte figurent en annexe : les deux en couleurs avec la chroniqueuse dans son village natal, elle-même habillée en bleu et blanc et tenant le magazine à la main devant la porte emblématique, contrastent avec celle en noir et blanc connotant l'ancienneté, qui illustre le récit sur Sidi Bou Saïd.

16. Cette formule a inspiré la communication touristique prônant la liberté du corps depuis les années 1970 . 
17. Dans la relation entre texte et image, Barthes (1964:44) distingue les fonctions d'ancrage (le texte téléguidant la lecture de l'image) et de relais (l'image apportant un complément ou une orientation au texte).

\section{RÉSUMÉS}

Cet article étudie l'impact de la crise politique sur les genres de discours promotionnels touristiques peu après le déclenchement du Printemps arabe. Nous analysons un corpus multimédiatique constitué de supports iconotextuels (c'est-à-dire composés de texte et d'image) et visant la promotion de deux pays arabophones méditerranéens : la Tunisie et l'Égypte.

D

eux lignes conductrices nous guident dans cette recherche. D'un côté, nous identifions une évolution générique des discours englobés sous la rubrique touristique qui se différencient entre documents de promotion proprement dite et documents de soutien. D'un autre côté, nous abordons

l'influence du contexte politique problématique sur la mise en place des démarches argumentatives, analysées selon un angle sémiolinguistique et pragmatique prenant en compte le verbal comme le visuel.

This article examines the impact of the political crisis on the kinds of promotional tourism discourses shortly after the outbreak of the Arab Spring. We analyze a multimedia corpus of iconotextual supports aimed at promoting two Arabic-speaking Mediterranean countries, Tunisia and Egypt. Two types of developments are examined. On the one hand, we identify a generic evolution of discourses encompassed under the tourism heading by distinguishing between promotional documents proper and supporting documents. On the other hand, we address the

influence of the problematic political context on the implementation of argumentative approaches, analyzed from a semiolinguistic and pragmatic angle taking into account the verbal and the visual.

\section{INDEX}

Mots-clés : tourisme arabe, post-crise, genre de discours, iconotextes, argumentation, séquences textuelles, actes de discours.

Keywords : arab tourism, post-crisis, gender of discourse, iconotext, argumentation, textual sequences, speech acts.

\section{AUTEUR}

\section{NABIL SUKKARI}

Université de Bourgogne Franche-Comté - ELLIADD 